\title{
Effect of Filler Mixture Ratio on Permeability of Multicomponent Soft Magnetic Composites
}

\author{
R. Dosoudil*, M. Ušáková, A. Grusková, J. Sláma \\ Faculty of Electrical Engineering and Information Technology, Ilkovičova 3, 81219 Bratislava, Slovakia \\ Composites with hybrid LiZnTi ferrite/FeSiAl alloy filler and PVC polymer matrix were synthesized by ther- \\ mal pressing process. The influence of the mixture ratio of hybrid filler on the frequency dispersion of complex \\ permeability of the composites has been studied. The dispersion of permeability varied from resonance type to \\ relaxation one with the configuration change from LiZnTi/PVC composite to $\mathrm{FeSiAl} / \mathrm{PVC}$ one and was mainly \\ due to the magnetic resonance phenomena such as domain wall and spin precession resonance and due to the eddy \\ current effect.
}

DOI: $10.12693 /$ APhysPolA.126.404

PACS: 75.50.Gg, 75.50.Bb, 76.50.+g, 75.78.-n, 72.80.Tm

\section{Introduction}

Recently, composite materials consisting of magnetic fillers in a host polymer matrix have attracted much attention due to their value for extensive applications at high frequency in industry, commerce and defense. Compared with ferrite fillers, metallic magnetic fillers have higher saturation magnetization and the Snoek's limit exists at a high frequency side $[1,2]$. However, their permeability decreases dramatically in $\mathrm{GHz}$ range since eddy currents induced by high frequency irradiation increase rapidly due to high electrical conductivity. Soft ferrite fillers are insulating materials, so the composites of soft ferrites and metallic magnetic materials can be better alternatives. The aim of this report is to synthesize LiZnTi/FeSiAl/PVC composites and analyze their frequency variation of complex permeability in the broad frequency range $(1 \mathrm{MHz}-6.5 \mathrm{GHz})$.

\section{Experimental}

LiZnTi ferrite of composition $\mathrm{Li}_{0.525} \mathrm{Zn}_{0.30} \mathrm{Ti}_{0.35}$ $\mathrm{Fe}_{1.825} \mathrm{O}_{4}$ (synthesized by a ceramic route at $1050{ }^{\circ} \mathrm{C} / 3.5 \mathrm{~h}$ in air) and a commercially available FeSiAl alloy (Kovohuty Co., Dolný Kubín, Slovakia) of composition $\mathrm{Fe}_{82.5} \mathrm{Si}_{15.0} \mathrm{Al}_{0.3}$ (the rest to balance present $\mathrm{Mn}, \mathrm{Cr}$ and $\mathrm{C}$ with amount less than $1 \%$ ) in bulk and also powder form were used as magnetic fillers. The particle size of fillers was controlled under $250 \mu \mathrm{m}$ for LiZnTi ferrite and under $160 \mu \mathrm{m}$ for FeSiAl alloy, respectively. The powder fillers were uniformly mixed with polyvinylchloride (PVC) at a constant total filler volume content of $60 \mathrm{vol} \%$. The mixture ratio of hybrid LiZnTi:FeSiAl filler was set to 1:0, 0.75:0.25, 0.50:0.50, 0.25:0.75, and 0:1. Ring shaped composite samples (outer diameter $7.9 \mathrm{~mm}$, inner diameter $3.2 \mathrm{~mm}$ and thickness of $1.8-2.5 \mathrm{~mm}$ ) were obtained by thermal pressing the ferrite/alloy/polymer mixture into a brass

*corresponding author; e-mail: rastislav.dosoudil@stuba.sk die under a pressure of $5 \mathrm{MPa}$ and a temperature of $145{ }^{\circ} \mathrm{C}$. Complex permeability was measured on final samples by a combined impedance/network analysis method. The dc electrical conductivity was determined using standard two-probe procedure.

\section{Results and discussion}

The plots of complex permeability $\mu=\mu^{\prime}-j \mu^{\prime \prime}$ versus frequency $f$ for bulk FeSiAl alloy and LiZnTi ferrite are shown in Fig. 1. The real part $\left(\mu^{\prime}\right)$ of $\mu$ in FeSiAl alloy is about 203 at a frequency of $100 \mathrm{~Hz}$ and then after a small rise it drops sharply to a very small value. The imaginary part $\left(\mu^{\prime \prime}\right)$ reaches a peak value of 224 at $f_{1}=316 \mathrm{~Hz}$. On the other hand, the $\mu^{\prime}$ in LiZnTi ferrite has a constant value of 92 at first then attains a certain growth followed by a steep decline. The $\mu^{\prime \prime}$ showed two peak values, the first about 55 at $f_{1}=16 \mathrm{MHz}$ and the second around 9 at $f_{2}=655 \mathrm{MHz}$. A small negative value of $\mu^{\prime}$ was observed between 2.3 and $6 \mathrm{GHz}$ as depicted in the inset of Fig. 1.

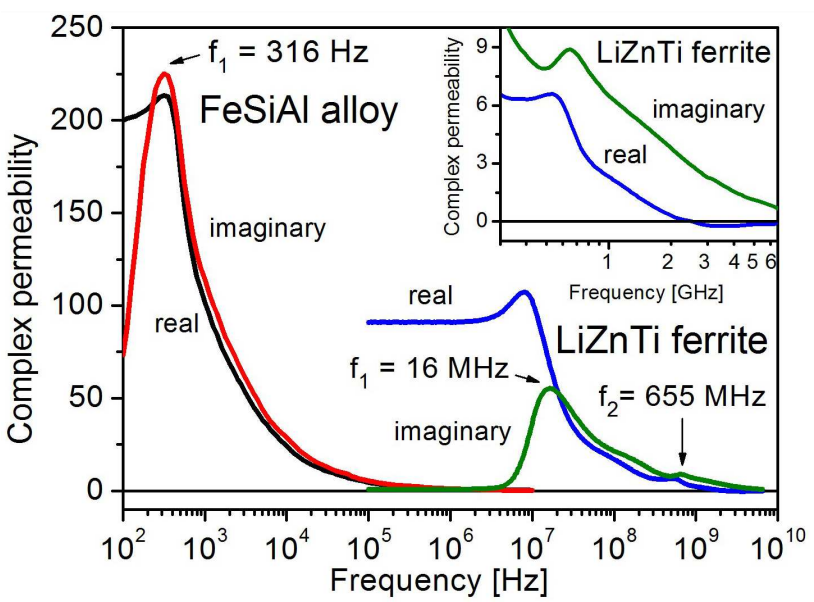

Fig. 1. Complex permeability vs. frequency for bulk FeSiAl alloy and LiZnTi ferrite. The inset shows the detail for LiZnTi ferrite in $\mathrm{GHz}$ frequency region. 
In magnetic materials, the frequency variation of permeability is related with its dispersion and can be explained by magnetic resonance and eddy current effect. The former is due to the domain wall oscillation at low frequency $\left(f_{1}\right)$ and the spin precession or natural ferromagnetic resonance at higher frequency $\left(f_{2}\right)$. The latter is caused by formation of magnetic moments due to eddy currents flowing on the surface of metallic material. Because FeSiAl alloy is conductive (with dc conductivity $\sigma_{d c}=7.9 \times 10^{5} \mathrm{Sm}^{-1}$ ), the eddy current effect is responsible for the abrupt decrease of permeability with frequency. The eddy currents bring about the drop of an effective volume contributing to the permeability of material thanks to the fall of the skin depth $\delta \approx \sqrt{1 /\left(\pi f \sigma_{d c} \mu_{o} \mu^{\prime}\right)}[3]$. Since the abrupt decrease of $\mu^{\prime}$ can happen above the frequency, at which $\delta$ starts to be smaller than the sample thickness $(d \approx 2 \mathrm{~mm})$, it is possible to estimate this frequency, $f_{\text {eddy }}$, as $f_{\text {eddy }} \approx$ $1 /\left(\pi \sigma_{d c} \mu_{o} \mu^{\prime} d^{2}\right)$. Calculated value of $f_{e d d y} \approx 396 \mathrm{~Hz}$ for FeSiAl alloy is close to the peak frequency $f_{1}=316 \mathrm{~Hz}$ of $\mu^{\prime \prime}-f$ dependence. In case of LiZnTi ferrite, the low frequency maximum of $\mu^{\prime \prime}$ (at $f_{1}$ ) corresponds to the domain wall resonance while the high frequency one (at $\left.f_{2}\right)$ indicates the natural ferromagnetic resonance. The negative value of $\mu^{\prime}$ in the inset of Fig. 1 originates from the magnetic resonance owing to the internal magnetic anisotropy field. Note that due to the low conductivity of LiZnTi ferrite $\left(\sigma_{d c}=5 \times 10^{-12} \mathrm{Sm}^{-1}\right)$, the contribution of eddy current effect can be neglected.

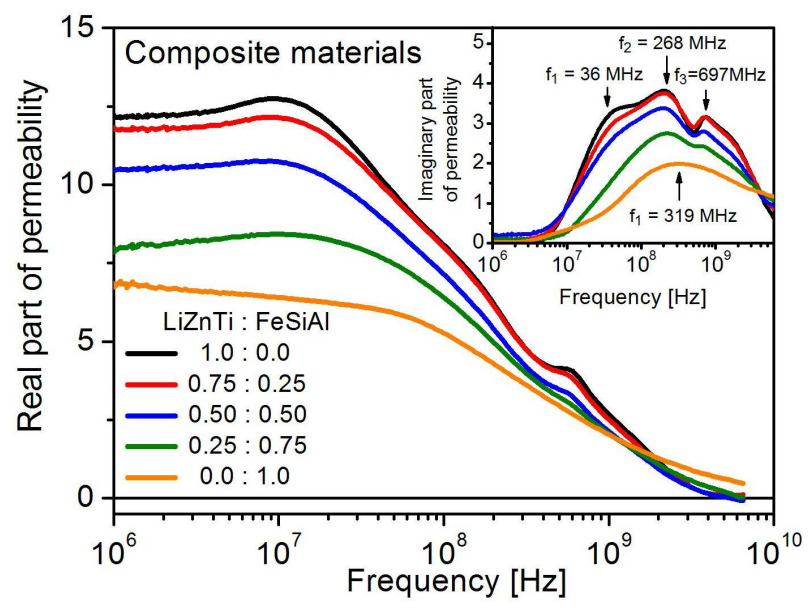

Fig. 2. Real part and imaginary part (inset) of complex permeability vs. frequency for synthesized composites.

The $\mu-f$ plots of LiZnTi/FeSiAl/PVC composites can be found in Fig. 2. The $\mu^{\prime}$ at low frequency $(1 \mathrm{MHz})$ in composite samples decreases from 12.2 for $\mathrm{LiZnTi} / \mathrm{PVC}$ composite to 6.9 for $\mathrm{FeSiAl} / \mathrm{PVC}$ one. The type of frequency dispersion of $\mu$ changes from resonance one, reached in $\mathrm{LiZnTi} / \mathrm{PVC}$ sample, to relaxation one ob- served in $\mathrm{FeSiAl} / \mathrm{PVC}$ composite. The ferrite/polymer composite exhibited three resonance maxima in $\mu^{\prime \prime}-f$ dependence as can be seen in the inset of Fig. 2: the first at $f_{1}=36 \mathrm{MHz}$ (which corresponds to $f_{1}$ in LiZnTi ferrite), the second at $f_{2}=268 \mathrm{MHz}$ and the third at $f_{3}=697 \mathrm{MHz}$ (which is near $f_{2}$ in LiZnTi ferrite). On the other hand, the metal alloy/polymer composite achieved only one peak in $\mu^{\prime \prime}-f$ plot at $f_{1}=319 \mathrm{MHz}$ and it is assumed that this peak is due to the coincidence of both magnetic resonances (domain wall and natural ferromagnetic one). In this composite, the influence of eddy currents cannot be neglected since the largest particle size $160 \mu \mathrm{m}$ is much higher than the skin depth $\delta \approx 2.3 \mu \mathrm{m}$ at $300 \mathrm{MHz}$ and approximated value of $f_{\text {eddy }} \approx 458 \mathrm{MHz}$ is slightly behind the $f_{1}=319 \mathrm{MHz}$. It should be noted that composites with the mixture ratios of hybrid LiZnTi:FeSiAl filler 0.75:0.25, 0.50:0.50 and 0.25:0.75 reached two maximum values in $\mu^{\prime \prime}-f$ plots: the first is between $f_{2}$ of LiZnTi/PVC sample and $f_{1}$ of $\mathrm{FeSiAl} / \mathrm{PVC}$ one, and the second is around $f_{3}$ of LiZnTi/PVC composite. Unlike bulk samples, the differences exhibited in $\mu-f$ dependences for composites are direct consequence of demagnetizing effects in filler particles: $\mu^{\prime}$ decreased and resonance frequencies $f_{1}, f_{2}$ shifted towards higher values due to the contribution of demagnetizing field. Moreover, the change of the filler mixture ratio in composites led to the alternation of magnetocrystalline anisotropy and accordingly also the values of $\mu^{\prime}$ and $f_{1}, f_{2}$.

\section{Conclusions}

The LiZnTi/FeSiAl/PVC composites with variable hybrid filler mixture ratio were prepared by thermal pressing method and the frequency variation of complex permeability was measured and analyzed. Whereas bulk samples (LiZnTi ferrite and FeSiAl alloy) showed the resonance type of permeability dispersion, the composites exhibited both the resonance and relaxation one. The dispersion of permeability was due to the domain wall and natural ferromagnetic resonance phenomena. The eddy currents affected the permeability too, especially in samples with higher content of conductive magnetic filler.

\section{Acknowledgments}

This work was supported by Slovak VEGA agencygrant No. 1/1163/12 and by Slovak Research and Development Agency (APVV) under the contract No. APVV0062-11.

\section{References}

[1] N. Joseph, M.T. Sebastian, Mater. Lett. 90, 64 (2013).

[2] R. Dosoudil, M. Ušáková, J. Sláma, Chem. Listy 105, 432 (2011).

[3] A.H. Taghvaei, H. Shokrollahi, K.Janghorban, J. Magn. Magn. Mater. 321, 3926 (2009). 